\title{
La Sociología en Venezuela. Una mirada a la disciplina y profesión desde dos generaciones.
}

\author{
Mari Carmen Pérez R.* \\ Carmen Teresa García*
}

\begin{abstract}
Resumen
El trabajo que presentamos, es parte de una investigación más amplia sobre la Sociología venezolana ayer y hoy ${ }^{1}$. En esta oportunidad, vamos a hacer referencia al análisis de algunos resultados parciales de la investigación:

En primer lugar, a un análisis cualitativo de la definición del egresado/a a partir del perfil académico plasmado en documentos como los planes de estudio de las Escuelas de Sociología de universidades venezolanas, la publicación referida a oportunidades de estudios en las Instituciones de Educación Superior en Venezuela del Consejo Nacional de Universidades (CNU), el Manual Descriptivo de Clasificación de Cargos de la Oficina Central de Personal, y la Ley de Colegiación.

En segundo lugar, se presentarán diez (10) estudios de caso de sociólogos/as que se han destacado en el desarrollo de esta disciplina como profesionales en las universidades (docentes e investigadores/as) y en la administración pública y privada. Estos profesionales opinaron sobre el Objeto de Estudio de la Sociología; Relación de las Escuelas de Sociología, el Mercado de Trabajo y el Perfil de Egreso; su vinculación con otras disciplinas y los desafíos de la Sociología como disciplina y profesión.

En tercer lugar, un análisis cuanti-cualitativo de los datos obtenidos de una encuesta aplicada a 62 sociólogos/as egresados(as) en la década de los 90, referida a la caracterización del profesional de Sociología y su quehacer como sociólogo/a (datos demográficos, estudios realizados,
\end{abstract}

\footnotetext{
Socióloga Venezolana, Universidad Central de Venezuela (UCV), Caracas-Venezuela. Magíster en Ciencias Políticas, Profesora e Investigadora, Departamento de Preventiva y Social de la Universidad de Los Andes (ULA), Mérida-Venezuela. Teléfono of. 0058 274 2402382 / 2390 Correo Electrónico: maricarmenperez_reyes@yahoo.com maricar@ula.ve

** Socióloga Venezolana, Universidad Católica Andrés Bello (UCAB), CaracasVenezuela. Doctorado de 3er. Ciclo en Estudios de América Latina, La Sorbona, ParísFrancia. Profesora e Investigadora del Departamento de Antropología y Sociología de la Facultad de Humanidades y Educación, Universidad de Los Andes (ULA), Mérida-Venezuela. Telefax 00582742401830 Correo Electrónico: ctgarcia@ula.ve tgr275us2001@yahoo.es

1 Agradecemos alCDCHT-ULA, instancia que financió estos proyectos deinvestigación (Códigos O-122-05-09-B y H-881-05-09-B).
} 
situación laboral), y finalmente, algunas conclusiones que se desprenden de esta investigación como la existencia de un desencuentro entre el perfil académico y el profesional, una invisibilización de la disciplina y la profesión en el mercado de trabajo en tiempos de globalización, y queremos dejar abierta la discusión del desafío que tiene la Sociología como disciplina y profesión ante la transdisciplinariedad que la invisibiliza y/o la desaparece como tal.

Palabras clave: Perfil de egreso, Mercado de Trabajo, Sociología, Venezuela.

\begin{abstract}
This paper is part of a broader research on the Venezuelan sociology in previous times and today. In this chance, we will make reference to the analysis of some partial results of the research:

In the first place, we refer to a qualitative analysis of the definition of the graduate starting from the academic profile stated in documents such as the curricula of the Sociology Schools in Venezuelan Universities, the publication referred to the studies opportunities in the Universities in Venezuela emitted by the National Universities Bureau (CNU for its name in Spanish), from the Descriptive Manual of Jobs Classification from the Main Personnel Office and the Collegiate Law.

-In the second place, we refer to the results of ten (10) case studies of Sociologists that have highlighted the development of this discipline as professionals in colleges (researches and faculties) and in the public and private administration. These professionals gave their opinions on the object of study of Sociology, relationship among the sociology schools, job offers in the market and the graduate profile; the links of sociology to other disciplines and the challenges of Sociology as a discipline and as a profession.

In the third place, a qualitative-quantitative analysis of some data obtained from a survey to 62 sociologists graduated in the 90 s, referred to the characterization of the sociology professional and his or her job as a sociologist (demographic information, academic information, working situation), and finally some conclusions from this research such as the existence of a mismatching between the academic profile and the professional one, a disappearance of the discipline and the profession from the job offers in the market in these globalization times and we want to open a discussion on the challenges of the Sociology as discipline and as a profession in front of the multidisciplinary environment that is making it disappear as such.
\end{abstract}

Key words: Graduate profile, Job offers, Sociology, Venezuela. 


\section{Introducción}

Este trabajo es parte de una investigación aplicada más amplia en curso, titulada "La Sociología Venezolana Ayer y Hoy". Por tal razón, este trabajo sólo presenta un análisis cuanti-cualitativo de algunos resultados parciales que pretenden aportar elementos para la discusión sobre el perfil y el quehacer de los(as) sociólogos(as) y la Sociología en Venezuela. ${ }^{2}$

En este trabajo abordamos el "Perfil de Egreso", a partir de dos dimensiones: el perfil profesional y el académico. De acuerdo con Glazman (1994:66-69, citado en Casarini, 1999:95) el "Perfil de Egreso" se constituye con los conocimientos, destrezas, habilidades, actitudes y valores requeridos para satisfacer las necesidades éticas, políticas y económicas en los ámbitos laboral y social. Se concreta en tareas, funciones, actividades y acciones susceptibles de llevarse a cabo por parte del egresado de determinado nivel.

Consideramos que en la actualidad ambos perfiles no pueden definirse separadamente. De acuerdo con Víctor Guédez (2000), el perfil académico-profesional es un conjunto de orientaciones, disposiciones, conocimientos, habilidades y destrezas, las cuales son deseables y factibles para obtener un grado académico determinado y ejercer labores ocupacionales en el área de dicho título. Si bien sabemos que el perfil profesional destaca la formación técnica determinada por el mercado laboral, mientras

2 De los cuestionarios aplicados en Venezuela (2002) , sólo se analizan para el presente estudio diez casos de profesionales de sociología (cinco sociólogas y cinco sociólogos del país), egresados/as de diferentes Escuelas de Sociología, Universidad Central de Venezuela, Caracas (UCV), Universidad Católica Andrés Bello, Caracas (UCAB), Universidad del Zulia, Maracaibo-Estado Zulia (LUZ), Universidad Nacional Experimental de Los Llanos Occidentales "Ezequiel Zamora", Barinas-Estado Barinas, (UNELLEZ), de los cuales tres se desempeñan actualmente en la administración privada y siete en las universidades respectivas del país como profesores(as) e investigadores(as) de diversas especialidades. En este artículo también se presentan algunos de los resultados de las encuestas aplicadas en Venezuela (2000-2001), a 62 profesionales de Sociología (24 sociólogos y 38 sociólogas) egresados(as) de la UCV, UCAB, LUZ y UNELLEZ que se desempeñan en las universidades como docentes e investigadores y en diferentes cargos $u$ oficios en la administración pública y privada. 
que el académico refleja una formación eficiente, racional, crítica y ética, consideramos que ambos perfiles deben estar integrados para garantizar la educación integral del futuro egresado. Tanto el perfil profesional como el académico no pueden ser definidos en forma aislada, ya que ambos cubren las necesidades de áreas específicas del ser humano: el trabajo y la formación del espíritu. Ambas dimensiones son influidas por los paradigmas dominantes determinados en la estructura social, por lo que no pueden ser excluyentes, ya que constituyen el perfil del egresado de una carrera en particular. Ambos perfiles inciden en la definición de los procesos educativos, se nutren (saber, saber-hacer, teoría-praxis) y constituyen un marco para el diseño del currículo o plan de estudio de determinada carrera.

Glazman (1994) plantea que el perfil de egreso parte de un enfoque que presenta dos requerimientos, que varían según las condiciones histórico-sociales: a) Los requerimientos de práctica, que se integran con los servicios requeridos por los sectores público y privado, en función de condiciones sociales, económicas, políticas, entre otras, y b) Los requerimientos académicos de la formación definidos por una institución educativa cuyo compromiso social, disponibilidad y límites corresponden a un ámbito que difiere de la producción y rebasa al mercado de trabajo. Entre estos dos requerimientos se establece una relación dialéctica: la formación académica incide en la práctica y ésta sugiere parte de la actualización del currículo o plan de estudios. En este sentido, las instituciones educativas influyen en las transformaciones de la práctica mediante la difusión de los cambios del conocimiento, las formas de transmisión y necesidades sociales en áreas específicas (Dimensión Académica); a su vez, la práctica sustenta la formación mediante el intercambio técnico y la disposición de recursos (Dimensión Profesional). Ambas dimensiones se sustentan y deben estar incluidas en el currículo, el cual contribuirá en el/la futuro/a egresado/a. 
Otras de las definiciones que están presentes en el análisis, es el de Mercado de Trabajo, que es donde se establecen las transacciones entre grupos particulares de personas (agentes que ofrecen y demandan bienes y servicios específicos), asignando así valor y fijación al precio de su trabajo, expresados generalmente en términos monetarios, prevaleciendo la competencia profesional. El trabajo es el bien que se ofrece y demanda, siendo oferentes los y las empleados/as y el personal empleador el o la demandante (empresas privadas y públicas), (Pérez R., 2001).

Las dos definiciones anteriores las asumimos teniendo presente que la investigación se ha elaborado en un contexto de una sociedad capitalista en tiempos de globalización asimétrica, que influye en el orden mundial y en el desarrollo de los países. El proceso de globalización ${ }^{3}$ cubre un campo mucho más amplio y complejo que el económico, pues incorpora también cambios en los ámbitos político, ideológico, cultural, tecnológico, socioeducativo, entre otros, que generan un profundo impacto no sólo en el tipo de políticas económicas, sociolaborales (flexibilidad laboral) y socioeducativas (privatización y la "calidad, productividad y competitividad" (políticas de ajuste estructural en Venezuela, en la década de los noventa), sino también en la Sociología como disciplina y profesión, su acción social y el quehacer sociológico de los profesionales, lo cual constituye nuestra pre/ocupación.

\section{Los Planes de Estudio de las Escuelas de Sociología de las Universidades Venezolanas en la década de los 90}

La evaluación de la formación de los estudiantes de la carrera de Sociología y su desempeño en la práctica profesional se puede

3 Apartir de la década de los noventa, implica la simultaneidad de una serie de complejos procesos, en la cual se genera un conjunto de redefiniciones y reestructuraciones a nivel del proceso de acumulación de capital, las instituciones, especialmente el Estado, quien establece nuevas formas de regulación económica, política, social y cultural, plantean un mundo más interconectado e interdependiente, pero con un carácter asimétrico y excluyente en la mayoría de los casos (Santana, 1997). 
La Sociología en Venezuela. Una mirada a la disciplina y profesión desde dos generaciones

comprender a partir del contenido temático de cuatro (4) Planes de Estudio de las Escuelas Profesionales de Sociología de diferentes Instituciones de Educación Superior en Venezuela durante la década de los noventa: Escuela de Sociología de la Universidad Central de Venezuela (UCV), Caracas (Región Capital); Escuela de Ciencias Sociales (Sociología) de la Universidad Católica Andrés Bello (UCAB), Caracas (Región Capital); Escuela de Sociología de la Universidad del Zulia (LUZ), Maracaibo-Estado Zulia (Región Zuliana); Escuela de Sociología del Desarrollo de la Universidad Nacional Experimental de Los Llanos Occidentales "Ezequiel Zamora" (UNELLEZ), Barinas-Estado Barinas (Región de Los Andes). ${ }^{4}$ (Véanse Cuadros $\mathrm{N}^{\circ} 1$ y N²)

\section{Plan de Estudios de la Escuela de Sociología de la Universidad Central de Venezuela (UCV), Caracas (Región Capital)}

El Plan de Estudios se diseñó tomando en cuenta dos exigencias para la formación del científico social: Una referida a la preparación básica y otra relativa a las líneas de escogencia de perfiles curriculares. En la primera se pretende impartir al estudiante los conocimientos básicos de carácter teórico-metodológico en función del desarrollo y avances de la teoría y la investigación en las ciencias sociales; mientras en la segunda se desea proporcionar un área electiva de trabajo, en la cual los estudiantes podrán profundizar en temáticas y campos de conocimientos específicos que les permitirán ir orientando su formación con miras al ejercicio profesional. En esta área serán fundamentales las líneas curriculares ofrecidas por cada uno. A través de las mismas no se pretende que el estudiante adquiera una especialización a nivel del pregrado, sino ofrecerle la posibilidad de profundizar en campos

4 La Universidad de Oriente (UDO) sí tiene una Escuela de Sociología, sin embargo, esta no está incluida dentro de las instituciones que formaron a los profesionales de sociología entrevistados del presente estudio. La ULA, LUZ, UNELLEZ son Instituciones de Educación Superior de Venezuela de diferentes regiones que están ubicadas en el Occidente de Venezuela, a diferencia de la UCV y UCAB que están ubicadas en la Región Capital (Caracas). 
más particulares de conocimiento más allá de una formación común a todo el estudiantado de la Escuela.

Cuadro $n^{\circ} 1$. Planes de estudio de las escuelas de sociología en Venezuela década de los 90

\begin{tabular}{|l|c|c|c|c|}
\hline Asignaturas & UCV & LUZ & UNELLEZ & UCAB \\
\hline Introducción al Estudio de la Sociedad & $\mathrm{X}$ & & $\mathrm{X}$ & \\
\hline Sociedad de Masas & & & & $\mathrm{X}$ \\
\hline Introducción a la Sociología & & $\mathrm{X}$ & & \\
\hline Sociología I, II, III & & & & $\mathrm{X}$ \\
\hline Corrientes Sociológicas Contemporáneas & & & & $\mathrm{X}$ \\
\hline Teoría Social I, II, III y IV & $\mathrm{X}$ & $\mathrm{X}$ & $\mathrm{X}$ & \\
\hline Cambio Social en Venezuela I y II & & & & $\mathrm{X}$ \\
\hline Estratificación y Segmentación Social & & & & $\mathrm{X}$ \\
\hline Dinámica y Cambio Social & & & & $\mathrm{X}$ \\
\hline Introducción a la Planificación & & & $\mathrm{X}$ & \\
\hline Teoría General de la Planificación & $\mathrm{X}$ & & & \\
\hline Planificación Social & & $\mathrm{X}$ & $\mathrm{X}$ & $\mathrm{X}$ \\
\hline Introducción al Trabajo Intelectual & & & $\mathrm{X}$ & \\
\hline Artesanía Intelectual & & $\mathrm{X}$ & & \\
\hline Metodología I, II, III & $\mathrm{X}$ & $\mathrm{X}$ & $\mathrm{X}$ & $\mathrm{X}$ \\
\hline Talleres de Investigación I, II, III, IV, V, VI & $\mathrm{X}$ & & & \\
\hline Introducción al Conocimiento Científico & & & $\mathrm{X}$ & $\mathrm{X}$ \\
\hline Epistemología & & & $\mathrm{X}$ & \\
\hline Introducción a la Filosofía & & & $\mathrm{X}$ & \\
\hline Filosofía Social y Política & & & $\mathrm{X}$ & \\
\hline Ética Profesional & & & & $\mathrm{X}$ \\
\hline Legislación Social I y II & & $\mathrm{X}$ & $\mathrm{X}$ & \\
\hline Computación e Informática & & $\mathrm{X}$ & & $\mathrm{X}$ \\
\hline Matemáticas & & $\mathrm{X}$ & $\mathrm{X}$ & \\
\hline Estadística I, II y III & & & $\mathrm{X}$ & $\mathrm{X}$ \\
\hline Demografía & & & $\mathrm{X}$ & $\mathrm{X}$ \\
\hline Inglés & & $\mathrm{X}$ & $\mathrm{X}$ & $\mathrm{X}$ \\
\hline Comunicación & & $\mathrm{X}$ & $\mathrm{X}$ \\
\hline Lenguaje y Comunicación & & $\mathrm{X}$ & $\mathrm{X}$ \\
\hline Psicología & & & \\
\hline Psicología Social & & & $\mathrm{X}$ \\
\hline Procesos Socioculturales I y II & & & \\
\hline Geografía Humana & & & $\mathrm{X}$ \\
\hline Medio Ambiente & & & \\
\hline Ecología y Sociedad & & & \\
\hline Ecología y Desarrollo & & & \\
\hline Desarrollo Regional I y II & & & \\
\hline Estrategia de Desarrollo & & & \\
\hline Desarrollo Económico & & & \\
\hline
\end{tabular}


La Sociología en Venezuela. Una mirada a la disciplina y profesión desde dos generaciones - Mari Carmen Pérez R. y Carmen Teresa García

\begin{tabular}{|l|c|c|c|c|}
\hline Macroeconomía y Microeconomía & & & & $\mathrm{X}$ \\
\hline Agronomía Venezolana & & & $\mathrm{X}$ & \\
\hline Economía I, II y III & & $\mathrm{X}$ & $\mathrm{X}$ & \\
\hline Economía Agrícola & $\mathrm{X}$ & & & \\
\hline Economía Política I, y II & $\mathrm{X}$ & $\mathrm{X}$ & & $\mathrm{X}$ \\
\hline Teorías Políticas & & & $\mathrm{X}$ & \\
\hline Teoría y Política de Desarrollo & & & & $\mathrm{X}$ \\
\hline Teoría de la Organización Burocrática & & & $\mathrm{X}$ & $\mathrm{X}$ \\
\hline Sistemas Políticos Comparados & & & & $\mathrm{X}$ \\
\hline Democracia y Sociedad & & $\mathrm{X}$ & & \\
\hline Política Social & $\mathrm{X}$ & $\mathrm{X}$ & & \\
\hline Sistemas y Procesos Históricos Contemporáneos & & & $\mathrm{X}$ & $\mathrm{X}$ \\
\hline Historia Económica y Social I y II & $\mathrm{X}$ & $\mathrm{X}$ & $\mathrm{X}$ & $\mathrm{X}$ \\
\hline América Latina I y II & $\mathrm{X}$ & $\mathrm{X}$ & & \\
\hline Formación Social Venezolana I y II & & & & $\mathrm{X}$ \\
\hline Sociología del Conocimiento & & & & $\mathrm{X}$ \\
\hline Sociología de la Familia & & & & $\mathrm{X}$ \\
\hline Sociología de la Desviación & & & & $\mathrm{X}$ \\
\hline Sociología Urbana & & & & $\mathrm{X}$ \\
\hline Sociología Rural & & & & $\mathrm{X}$ \\
\hline Sociología Política & & $\mathrm{X}$ & & \\
\hline Orientación y Autodesarrollo & & & $\mathrm{X}$ & \\
\hline Deportes & $\mathrm{X}$ & $\mathrm{X}$ & $\mathrm{X}$ & $\mathrm{X}$ \\
\hline Pasantías /Prácticas & $\mathrm{X}$ & $\mathrm{X}$ & $\mathrm{X}$ & $\mathrm{X}$ \\
\hline Optativas o Electivas & & $\mathrm{X}$ & $\mathrm{X}$ \\
\hline Diseño y Evaluación de Proyecto & $\mathrm{X}$ & $\mathrm{X}$ & $\mathrm{X}$ \\
\hline Seminario - Proyecto de Tesis & $\mathrm{X}$ & & \\
\hline Cátedra-Taller I y II & $\mathrm{X}$ & $\mathrm{X}$ \\
\hline Tesis de Grado & & \\
\hline
\end{tabular}

FUENTE: Elaboración propia con información de los Planes de Estudio. Mérida, 2006.

\section{Perfil de egreso}

En relación al perfil de egreso, el/la sociólogo/a como profesional es un/a investigador/a que estudia la génesis, el desarrollo y la transformación de las relaciones sociales de las sociedades humanas. En este sentido, también interpreta y analiza las formas de organización social, los modos de vida, la formación jurídica y política, las manifestaciones ideológicas y culturales, las formas de producción de los bienes materiales que forman parte del tejido social (Véase Cuadro $N^{\circ} 1$ y $N^{\circ} 2$ ). 
La Escuela de Sociología imparte al estudiante los conocimientos teóricos y metodológicos sobre la disciplina sociológica y los/as orienta en su formación para el ejercicio profesional. Entre las áreas más relevantes de estudio abordadas por la Escuela de Sociología se encuentran aquellas relativas a la conceptualización de la sociedad, sus estructuras y desarrollos, abarcando además problemas específicos tales como la educación, la salud, la vivienda, el trabajo, la vida urbana y rural, y otros aspectos de gran importancia en la realidad social del país.

Los estudios de Sociología no sólo permiten adquirir y contribuir a producir un conocimiento cabal sobre los procesos que conforma la realidad social, sino que además hace posible el diseño y la planificación de estrategias de desarrollo y cambio social.

\section{Plan de Estudio de la Escuela de Ciencias Sociales -Carrera de Sociología- de la Universidad Católica Andrés Bello (UCAB), Caracas (Región Capital)}

En la Escuela de Ciencias Sociales se cursan tres carreras: Relaciones Industriales, Sociología y Trabajo Social. La distribución general de las materias se presenta de acuerdo a la organización de la Escuela de Ciencias Sociales: Un Ciclo Básico de dos años y los Ciclos Profesionales de Relaciones Industriales y de Sociología de tres años de duración (UCAB, Pensum de Estudios de la Escuela de Ciencias Sociales, 1993).

\section{Perfil de egreso}

El/la sociólogo/a es un/ científico/a de las relaciones sociales, de la estructura de la sociedad, de la dinámica y de la acción social. Además del manejo de la teoría e instrumentos sociológicos propios, debe desenvolverse apropiadamente con los rudimentos básicos de otras ciencias y disciplinas que lo complementan, tales como la economía, la psicología, la historia, la demografía, la estadística y la politología, que en su praxis se transforman en Sociología aplicada a campos específicos del quehacer humano. 
El/la sociólogo/a es un/a profesional que posee conocimientos y herramientas para procurar que la gente actúe efectivamente en el marco de programas racionalmente planificados. En otras palabras, el trabajo de un/a sociólogo/a está vinculado con las actividades gerenciales y de planificación en las políticas sociales. El/la egresado/a debe manejar técnicas de gerencia y planificación (Véase Cuadro $\mathrm{N}^{\circ} 1$ y N²).

El/la sociólogo/a egresado/a de la Escuela de Ciencias Sociales de la UCAB deberá esforzarse por desempeñarse como:1) Un pensador crítico capaz de traducir los hechos sociales y económicos observados en un conjunto de ideas interrelacionadas y sistematizadas. 2) Un científico, capaz de interpretar las observaciones organizadas y sistematizadas a través de diferentes vías, partiendo de teorías y doctrinas explicativas de la realidad social. 3) Una persona comprometida en la construcción de una sociedad mejor. Es decir, el/la sociólogo/a deberá suministrar constantemente una visión sistematizada e interpretada de los hechos, visión que deberá orientar las actuaciones de la sociedad política. En resumen, es un/a estudioso/a de la realidad social en sus múltiples manifestaciones, sociales, económicas, culturales, psicológicas, políticas y organizacionales. Es un/a profesional capaz de cumplir la función de catalizador/a y orientador/a de los procesos de cambio en la sociedad y de intervenir en ellos con acciones eficaces (UCAB, Pensum de la Escuela de Ciencias Sociales, 1993).

\section{Plan de Estudio de la Escuela de Sociología de La Universidad del Zulia (LUZ), Maracaibo; Estado Zulia (Región Zuliana)}

En relación a la estructura curricular del plan de estudios se adoptó un esquema de ciclos para ordenar la administración de los contenidos y actividades formativas, estableciéndose un ciclo inicial, uno intermedio y uno terminal. Cada uno de ellos constituye un período que se espera permitan de manera progresiva una profundización de los conocimientos adquiridos por el estu- 
diante, a la vez, la operacionalización de los mismos (LUZ, Plan de estudio de la Escuela de Sociología, 1995).

\section{Perfil de egreso}

El/a sociólogo/a es un científico/a de nivel universitario, con una formación teórica y metodológica de carácter general, apto para investigar problemas, relaciones, estructuras y procesos sociales, tanto globales como particulares. Ello le habilita para participar e intervenir de manera orgánica como investigador/a, analista y gerente social en los procesos societarios, con el objeto de contribuir al logro de un desarrollo humano y social, en armonía con la naturaleza, tanto a nivel nacional como internacional; todo ello realizado con responsabilidad y ética social. En función de lo enunciado, la Escuela de Sociología de la Universidad del Zulia, estructura su perfil de acuerdo a los siguientes roles (Véase Cuadro $\mathrm{N}^{\circ} 1$ y $\mathrm{N}^{\circ} 2$ ):

- Investigador: Este rol alude a las funciones, actividades, competencias y valores que soportan el proceso de creación, reelaboración y aplicación de conocimiento sociológico, con base en la formulación de propuestas teóricas y modelos metodológicos que requiere la investigación, así como la construcción de técnicas e instrumentos cuantitativos y cualitativos consustanciales a las exigencias de medición, evaluación e indagación de la realidad social.

- Analista: Este rol alude a las funciones, actividades, competencias y valores que permiten al profesional de la sociología, diagnosticar, evaluar y explicar realidades sociales, aplicando enfoques y modelos sociológicos.

- Gerente Social: Este rol representa la síntesis de todas aquellas funciones, actividades, competencias y valores que permiten al profesional de la sociología, diseñar, planificar, coordinar, ejecutar y evaluar políticas sociales y procesos de gestión de un modo eficaz, eficiente y efectivo. El rol de investigador/a es básico para todas las labores que pueda desarrollar el/la sociólogo/a, sin embargo, pareció conveniente asignarle el 
mismo peso a todos los roles, por considerar que ellos se entrecruzan en las actividades y contenidos que se desarrollan en cada cátedra.

\section{Plan de Estudio de la Escuela de Sociología del Desarrollo de la Universidad Nacional Experimental de Los Llanos Occidentales "Ezequiel Zamora" (UNELLEZ), Barinas; Estado Barinas (Región de Los Andes)}

El Programa se crea en el marco de la concepción de un modelo universitario cuyas características fundamentales se encuentran en su carácter regional, experimental y la concepción de sistema universitario. Todo ello, con el fin de desarrollar y dirigir programas profesionales a nivel técnico (productividad) y a nivel superior (docencia-investigación y planificación) para contribuir al Desarrollo Nacional, aprovechando los recursos humanos y naturales de la región.

Entre los objetivos centrales del Programa Sociología del Desarrollo se pueden mencionar los siguientes: 1) Diagnosticar e investigar la realidad social a nivel micro y macro, 2) Formular y evaluar planes y proyectos de desarrollo, 3) Participar en la ejecución de proyectos de desarrollo, y 4) Intervenir en la organización, capacitación y adopción de nuevas técnicas y patrones culturales. Prima aquí una concepción determinada de lo social que implica un manejo técnico-instrumental de su conocimiento, lo cual lleva implícita una búsqueda del desarrollo, como modelo deseado y paradigma dominante de esta época (UNELLEZ, Pensum del Programa Sociología del Desarrollo, 1995). (Véase Cuadro $\mathrm{N}^{\circ} 1$ y $\mathrm{N}^{\circ} 2$ ).

\section{Perfil de egreso}

El perfil del egresado se fundamenta en dos premisas básicas: 1) La necesidad de contar con un egresado que sea capaz de des- 
cribir, explicar y comprender el tejido social en sus múltiples y polifacéticas expresiones. Lo cual da cuenta de una sólida formación epistemológica y teórica-metodológica, y 2) La necesidad de contar con un egresado que sea capaz, a partir de la crítica, de incorporarse al proceso de transformación, reconsideración y nuevos planteamientos en torno a la complejidad presente en lo real-social, lo que implica una postura ética definida.

A partir de estas premisas básicas, el/la egresado/a del Programa Sociología del Desarrollo estará preparado/a para: 1) Investigar la complejidad y diversidad presente en lo real-social. Incorporando, entre otros, aspectos referidos a: Procesos de desarrollo económico globales y micro-procesos, Procesos sociales específicos que den cuenta de realidades puntuales, Procesos políticos donde se evidencian caracterizaciones del Estado, democracia, formas de gobierno, partidos políticos, nuevos actores políticos, etc. 2) Planificar en términos de las nuevas realidades presentes en el ámbito de lo real-social. En este sentido puede participar en actividades planificadoras de procesos sociales integrales/globales.

En el Cuadro $\mathrm{N}^{\circ} 1$ referido a los Planes de Estudio de Sociología se pueden apreciar las asignaturas que obedecen a ciertas categorías que van delineando el perfil del contenido curricular en los Planes de Estudio de cada una de las Escuelas de Sociología: la Investigación (Metodología, Planificación), las Teorías (Teorías Sociales, Sociológicas, Sociología en General y las Ciencias Sociales), lo Social (problemas sociales, políticas públicas, estructura social), lo social desde el punto de vista macrosocial (UCV y LUZ) y microsocial (UNELLEZ y UCAB), las Sociologías Aplicadas, las Ciencias Afines e Idiomas.

En el Plan de Estudios de la Escuela de Sociología de la UCV, se puede apreciar una gran carga temática en la categoría de Investigación, Teorías, Ciencias afines, y lo Social desde el punto de vista macrosocial. En el Plan de Estudios de la Escuela de So- 
ciología de LUZ, se destaca una carga temática en la categoría Teorías, Investigación y Ciencias Afines. En el Plan de Estudios de la UNELLEZ se puede observar que la carga temática se orienta hacia lo Teórico-Metodológico, lo social es definido desde un nivel micro y macro, un nivel técnico-productivo, planificación y evaluación de proyectos de desarrollo a nivel local, regional. En el Plan de Estudio de Sociología de la UCAB, se puede observar una gran carga temática en la categoría de Sociologías Aplicadas, lo Social a partir de la estructura de la sociedad, la dinámica y acción social y desde el punto de vista microsocial. A partir de los contenidos temáticos presentes en los cuatro planes de estudio se puede apreciar el perfil de egreso, el cual se corresponde con la formación esperada en cada una de las Escuelas de Sociología (Véase Cuadro $\mathrm{N}^{\circ} 1$ y $\mathrm{N}^{\circ} 2$ ).

Como bien se pudo apreciar en el panorama general antes descrito, la creación de las Escuelas de Sociología en Venezuela ha adquirido un papel primordial en la profesionalización de la Sociología, pues ellas constituyen los campos fundamentales en el ámbito sociológico. Sus orientaciones se expresan en los Planes de Estudio, los cuales a su vez, expresan los perfiles de los/as sociólogos/as. Estos perfiles académicos se corresponden con el perfil del egresado establecido por el Consejo Nacional de Universidades (CNU, 2000) (Véase Cuadro $\mathrm{N}^{\circ} 1$ y N²).

\section{El/la sociólogo/a estudia según (CNU-OPSU, 2002:182):}

"El origen, evolución e interrelación de los grupos humanos. Plantea problemas del comportamiento social, diagnostica sus causas y jerarquiza prioridades de solución. Planifica y programa estudios e investigación, determina métodos y procedimientos, elabora instrumentos para la recolección y tabulación de la información, analiza resultados y elabora conclusiones y recomendaciones. Realiza estudios y labores de planificación, tales como investigaciones integrales de las comunidades, estudios de recursos humanos, diagnósticos regionales, diagnósticos organizacionales, definiciones de políticas y programas sociales, polí- 
ticas de promoción. Reúne y presenta los datos, conclusiones y recomendaciones en forma que puedan ser de utilidad a la administración, industria, organizaciones sociales u otros organismos. Se especializa en sociología rural, urbana, del delito, de la salud, de la educación, de la cultura, de la comunicación y sociología industrial entre otras especialidades". ${ }^{5}$

Las Escuelas de Sociología, adquieren una gran importancia en la legitimación social de la Sociología, en la formación académica y profesional de los/as sociólogos/as lo que influye en muy buena parte la relación de los/as sociólogos/as con el mercado laboral. Por ello, la Ley de Ejercicio Profesional de la Sociología y la Antropología, establece en el Artículo $4^{\circ}$ del Título II. Del Ejercicio de la Sociología y la Antropología: ${ }^{6}$

“El campo del ejercicio profesional de la Sociología y la Antropología se constituye a partir de los roles delimitados en los planes de estudio de las escuelas de las universidades nacionales, experimentales o privadas, debidamente autorizadas o en los cursos de postgrado de Sociología y Antropología aprobados por los organismos competentes en la materia, y también de lo establecido en los convenios con los institutos educativos o de investigación debidamente reconocidos y acreditados de otros países o con organismos internacionales con los cuales existen esos con-

5 Para mayor información sobre la carrera de Sociología en Venezuela que ofrecen las Instituciones de Educación Superior, véase Consejo Nacional de Universidades (CNU), Oficina de Planificación del Sector Universitario (OPSU), 2002. Oportunidades de Estudio en las Instituciones de Educación Superior de Venezuela, Caracas, Venezuela.

6 Véase Título I. Disposiciones Generales. Artículo $1^{\circ}$, de la Ley de Ejercicio Profesional de la Sociología y Antropología: "Esta Ley tiene por objeto la definición y delimitación del campo profesional de actividades relacionadas con el ejercicio de la Sociología y Antropología que requieran o estén fundamentadas en el conocimiento y empleo de teorías, metodologías, técnicas y aplicaciones prácticas propias de las ciencias sociológicas y antropológicas, sin desmedro de los nuevos avances científicos y de la participación de carácter interdisciplinario o aquellos que señalan la dinámica social e institucional. Asimismo facilitar el mejoramiento socioeconómico de los profesionales de la Sociología y la Antropología en el marco de lo establecido por la Constitución". (Gaceta Oficial de la República de Venezuela, Año CXXV-Mes IX, Caracas, martes 23 de junio de 1998, No5.239 Extraordinario. Ley de Ejercicio Profesional de la Sociología y Antropología). 
venios; de lo establecido en el Manual de Descripción de Cargos de la Oficina Central de Personal de la Presidencia de la República, de las definiciones del Diccionario Nacional de Ocupaciones del Ministerio del Trabajo en lo que corresponde a Sociólogos y Antropólogos y el Clasificador de Ocupaciones y Oficios de la Organización Internacional del Trabajo".

La Sociología es una disciplina ampliamente diversificada, presente en múltiples áreas del conocimiento, por lo que la descripción del perfil y del cargo del profesional de Sociología es definida a partir de la amplitud de áreas en las cuales ha incursionado, y de la competencia en actividades en diferentes disciplinas. Sin embargo, el Estado venezolano a través de la Oficina Central de Personal (OCP, 1980 y 1990) ha caracterizado y definido el trabajo y las tareas de los/as sociólogos/as y las implicaciones para la reproducción de la ideología tecnicista, instrumentalista y metodologizante del modelo de sociología profesionalizada.

La labor del profesional de Sociología puede derivarse de la descripción de cargos ofrecida en el Manual Descriptivo de Clasificación de Cargos de la OCP (1980 y 1990), la cual recoge algunas de las funciones más importantes asignadas al sociólogo/a que trabaja en la Administración Pública: Planificación, Coordinación, Evaluación y Supervisión, Administración de Personal, Gerencia social, Gerencia en Recursos Humanos, entre otras funciones. En el Cuadro $\mathrm{N}^{\circ} 2$, podemos apreciar que en tales funciones definidas por OCP (1980 y 1990), los/as sociólogos/as ocupan un espacio como profesionales al servicio del Estado y, en consecuencia, están sometidos a la organización burocrática de la profesión a partir de los criterios fijados por el Manual de Clasificación de Cargos, instrumento que opera como un delimitador del campo sociológico desde la perspectiva del sector oficial. Castro (1988) sostiene que el modelo profesionalizante de la Sociología es el que predomina en la representación que de la disciplina tiene el Estado como empleador. Esta visión del Estado se expresa a través de la prevaleciente interpretación de la So- 
ciología y de lo que hacen los/as Sociólogos/as, en términos de un utilitarismo, que a su vez se expresa, en la predominancia de las dimensiones técnico-metodológicas y operativas, empirismo, asumidas como campo de acción de la Sociología. Esta visiónrepresentación de lo sociológico presente en el Estado, y a la cual consideramos como el componente ideológico fundamental del modelo profesional implantado desde 1952, se constituye en un factor que contribuye con gran relevancia al reforzamiento de la misma ideología profesionalizante basada en el tecnicismo y en el metodologismo de los/as estudiantes de las Escuelas de Sociología del país. Sin embargo la orientación de la Sociología actual es pluriparadigmática, su ámbito de estudio es complejo, multidisciplinario y transdisciplinario, por lo que las funciones ejercidas por los/as sociólogos/as también son diversas y dependen en gran medida de las funciones y el nivel de compromiso con lo social que tengan los organismos donde ejercen los profesionales de la Sociología (Giddens, 2000).

En relación al mercado laboral, según CNU-OPSU (19882002), el trabajo que realiza el/la sociólogo/a es básicamente psíquico-verbal-social y se desarrolla, por lo general, en oficinas y al aire libre cuando debe participar en actividades comunitarias o de otro tipo inherentes a sus funciones específicas. El profesional de Sociología tiene oportunidades de trabajo en distintas áreas, entre algunas de ellas se pueden mencionar las siguientes:1) Docencia. En este aspecto existe un extenso campo de trabajo a todos los niveles educativos, principalmente en la enseñanza media y superior, 2) Investigación y Planificación. Los centros de investigación de las universidades, así como ciertos organismos privados y públicos, tales como: Ministerios: Salud y Desarrollo Social, Interior y Justicia, Planificación y Desarrollo, Educación, Cultura y Deportes, Fundacomún, entre otras instituciones, que emplean sociólogos/as que se dedican básicamente al estudio, análisis e interacción, a través de la planificación de la problemática socialeconómica, política, cultural, abarcando un extenso campo de la realidad social, 3) Libre Ejercicio de la Profesión. Algunos profesio- 
La Sociología en Venezuela. Una mirada a la disciplina y profesión desde dos generaciones - Mari Carmen Pérez R. y Carmen Teresa García

\section{nales de la Sociología trabajan por cuenta propia y ofrecen sus servicios especializados en empresas privadas y en condiciones de contratos.}

\section{Cuadro $\mathrm{N}^{\circ}$ 2. Perfil del/la egresado/a de sociología}

\begin{tabular}{|c|c|c|}
\hline INSTITUCIONES & DEFINICIÓN DE LA PROFESIÓN & ROLES \\
\hline UCV & $\begin{array}{l}\text { Científico Social e Investigador/a con formación } \\
\text { teórica y metodológica que estudia la génesis, el } \\
\text { desarrollo y las transformaciones sociales de las } \\
\text { sociedades humanas. } \\
\text { Analiza los procesos de la realidad social y } \\
\text { planifica estrategias de desarrollo y cambio } \\
\text { social. }\end{array}$ & $\begin{array}{l}\text { Investigador } \\
\text { Analista } \\
\text { Planificador }\end{array}$ \\
\hline UCAB & $\begin{array}{l}\text { Científico de las relaciones sociales, de la } \\
\text { estructura de la sociedad, de la dinámica y } \\
\text { acción social. }\end{array}$ & $\begin{array}{c}\text { Planificador } \\
\text { Gerente Social }\end{array}$ \\
\hline LUZ & $\begin{array}{l}\text { Científico con una formación teórica y } \\
\text { metodológica de carácter general apto para } \\
\text { investigar problemas, relaciones, estructuras } \\
\text { y procesos sociales, tanto globales como } \\
\text { particulares. }\end{array}$ & $\begin{array}{l}\text { Investigador } \\
\text { Analista } \\
\text { Gerente Social }\end{array}$ \\
\hline UNELLEZ & $\begin{array}{l}\text { Egresado capaz de describir, explicar y } \\
\text { comprender el tejido social. Investigar la } \\
\text { complejidad y diversidad en lo real-social y } \\
\text { planificar en términos de las nuevas realidades } \\
\text { sociales. }\end{array}$ & $\begin{array}{l}\text { Investigador } \\
\text { Planificador }\end{array}$ \\
\hline $\mathrm{CNU}$ & $\begin{array}{l}\text { Estudia el origen, evolución e interrelación } \\
\text { de los grupos humanos. Planifica y programa } \\
\text { estudios e investigación, determina métodos } \\
\text { y procedimientos, elabora instrumentos para } \\
\text { la recolección y tabulación de la información, } \\
\text { analiza resultados y elabora conclusiones y } \\
\text { recomendaciones. }\end{array}$ & $\begin{array}{c}\text { Docente } \\
\text { Analista } \\
\text { Planificador } \\
\text { Investigador } \\
\text { Gerente Social }\end{array}$ \\
\hline $\mathrm{OCP}$ & $\begin{array}{l}\text { Ocupan un espacio como profesionales al } \\
\text { servicio del Estado (Organización burocrática- } \\
\text { administrativa). } \\
\text { A partir de la estratificación de cargos se } \\
\text { establecen cuatro niveles jerárquicos: Sociólogo } \\
\text { Jefe, Sociólogo I, II y III. }\end{array}$ & $\begin{array}{l}\text { Planificador } \\
\text { Coordinador } \\
\text { Evaluador } \\
\text { Supervisor } \\
\text { Gerente Social } \\
\text { de Recursos } \\
\text { Humanos }\end{array}$ \\
\hline
\end{tabular}

FUENTE: Elaboración propia. Mérida, 2006. 


\section{Una mirada a la Sociología como Disciplina desde profesionales destacados. Resultados de una Investigación Cualitativa}

A continuación se presentan los resultados de un cuestionario aplicado, a diez profesionales de sociología, egresados/as de diferentes Escuelas de Sociología de los Institutos de Educación Superior de Venezuela que se han destacado en el desarrollo de esta disciplina en las universidades, en docencia-investigación y en la administración pública y privada, sobre el objeto de estudio de la Sociología; relación de las Escuelas de Sociología, el mercado de trabajo y el perfil de egreso; su vinculación con otras disciplinas y los desafíos de la Sociología como disciplina y profesión.

\section{a. Objeto de estudio de la Sociología}

Cuando nos acercamos a la realidad social como lo plantean Infestas y Lambea (1997) podemos hacerlo a través de tres niveles: el social (referido a los acontecimientos que una sociedad vive y que son análogos en todos los grupos humanos, pero debido a factores internos y externos que intervienen le confieren una originalidad indiscutible), el societal (referido al mundo de las significaciones que cada sociedad atribuye a los diversos hechos sociales. Esta interpretación incluye la elaboración simbólica del hecho en el que se enfatiza o se valoran algunos rasgos y que define su relevancia para el grupo o la sociedad en cuestión), y el sociológico (referido a la teorización sobre esta realidad social que tiene como referencia simbólica inevitable la interpretación que sus protagonistas hacen). Si consideramos pertinente esta distinción, también pudiera explicarnos el condicionamiento de lo societal sobre lo sociológico y que éste no se limita al objeto de estudio sino que también se halla presente en el sujeto que investiga y que es integrante de una sociedad y, en consecuencia, partí-

7 Las respuestas obtenidas de los diez profesionales entrevistados se procesaron utilizando la técnica (Zamitiz, 1995; Sabino, 1986) semejanza-diferencia a fin de agruparlas para facilitar el análisis cualitativo. 
cipe de sus interpretaciones y de sus significados. Asimismo nos explica por una parte, la presencia y los riesgos de etnocentrismo, sexismo, estratocentrismo y muchos otros "ismos" de los que no ha escapado la teoría sociológica y, por otra, las tendencias y énfasis predominantes en un momento histórico determinado, de nuestro quehacer como científicos/as sociales.

El carácter "objetivo" que presenta/n la/s sociedad/es, no puede ser considerado como algo previamente dado o establecido, sino como el resultado del dinamismo de los grupos sociales precedentes y presentes. La Sociología actual está llamada a confrontar con todas sus herramientas teórico-metodológicas, la explicación, análisis e interpretación de la crisis de la(s) sociedad/ es a través de sus múltiples aristas (económica, política, cultural, social, educativa, lo conocido, lo inesperado, lo incierto, etc.) y desde múltiples perspectivas de abordaje dada su creciente complejidad.

Esta realidad social que ha emergido en todas sus dimensiones, objeto de nuestra ciencia, es el producto de procesos sociales en los que intervienen factores muy diversos, que van desde una forma determinada de relación entre países a nivel mundial hasta las normas que han definido y definen las relaciones entre los/as integrantes de una sociedad o comunidad determinada. La idea de sociedad ya no es más un principio de unidad, es más bien el resultado de relaciones interdependientes pero asimétricas (en la mayoría de los casos) y de las grandes orientaciones (económicas, políticas, culturales, etc.) que son su entorno. Como bien lo señalan los/as entrevistados/as, el objeto de estudio de la Sociología se ha abierto a diversas modalidades para comprender el mundo social complejo desde distintos niveles, ya sea social, societal, sociológico, o desde un nivel microsociológico o macrosociológico de las realidades sociales. ${ }^{8}$

8 Véase el Artículo de Carmen Teresa García. 1998. "El quehacer de la sociología en tiempos de crisis", en FERMENTUM (Revista Venezolana de Sociología y Antropología) Grupo de Investigación en Socioantropología de la Ciudad (GISAC), 
Ritzer (1996) señala que la realidad social se ha analizado desde niveles microscópicos (micro-subjetivo-objetivo; ejemplo, definición social y conducta social) y macroscópicos (macro-subjetivo-objetivo; ejemplo, los hechos sociales), los cuales se cruzan gradualmente con otros formando parte del gran "continuum social" por lo que separarlos ha conllevado a diferenciaciones bien arbitrarias para poder analizar la realidad social, la cual constituye nuestro objeto de estudio.

A partir de las respuestas a la pregunta sobre el objeto de estudio de la Sociología, se resume que la Sociología es una disciplina ampliamente diversificada, presente en múltiples áreas del conocimiento, por lo que su objeto de estudio es definido a partir de la amplitud de campos en los cuales ha incursionado, y de la competencia en actividades de diferentes disciplinas. La Sociología es una de las disciplinas que integra las Ciencias Sociales, cuyo objeto de estudio permanece siendo complejo delimitar, dado que la misma se encuentra en constante vinculación, desde sus orígenes hasta nuestros días, con otras disciplinas o ciencias: Política, Economía, Historia, Derecho, Trabajo Social, Geografía, Psicología Social, Antropología, Educación, Filosofía, tan sólo por citar algunos de los campos de conocimientos que abordan temáticas afines al interés de la Sociología.

Entre otras reflexiones aportadas se destacó que aun cuando es difícil definir un objeto de estudio determinado del campo sociológico, se podría hacer el intento, como por ejemplo en el

Universidad de Los Andes, Año 8, N²1, Enero-Abril, Mérida, Venezuela. Este trabajo ofrece una muestra del quehacer de los/as sociólogos/as y antropólogos/as, a partir de resultados de sus investigaciones recientes. La muestra nos evidencia, primero la direccionalidad del "oficio del sociólogo", su práctica profesional real; segundo, la predominancia de producción de los/as académicos/as vinculada a las universidades. En este sentido, pudiera decirse que hay una marcada tendencia, sin desmerecerla, al abordaje macro-sociológico, como lo han mostrado otras investigaciones que se han realizado al respecto y, tercero, una vinculación muy estrecha a determinados campos del saber y en este caso concreto, a lo económico, a las ciencias políticas, de tal forma que se pierde generalmente los límites (felizmente a la hora actual) de las mismas, por la necesaria multidisciplinariedad y transdiscipliariedad que deberían mostrar las Ciencias Sociales del próximo milenio. 
siglo XIX lo hizo Durkheim, sin embargo, la situación actual es compleja y como bien lo planteó Simmel en una oportunidad, la Sociología debe ser entendida desde una perspectiva que coloca los objetos de las otras ciencias y disciplinas en su dimensión social en cuanto a producciones condicionadas por las relaciones humanas y su historicidad.

Entre otros aportes, en relación al objeto de estudio, señalan que la Sociología es la ciencia que se ocupa del estudio de las relaciones entre los sujetos sociales y las instituciones públicas y privadas en una sociedad determinada y en un período histórico concreto. Además, estudia las contradicciones que se suscitan entre los actores sociales, sus demandas, las estructuras sociopolíticas, el modo de producción, la cultura y las relaciones internacionales.

La Sociología también estudia y analiza, desde una perspectiva crítica, histórica e integrada, los procesos, actores e instituciones que desarrollan teorías y prácticas en sociedades determinadas. Además del objeto general, la Sociología dirige sus estudios sobre áreas que le son ciertamente propias. La Sociología estudia las características comunes de todos los fenómenos sociales. Estudia las relaciones que hay entre los distintos fenómenos, las cuales no pueden ser abordadas a cabalidad por las ciencias sociales. También configura un objetivo específico del estudio de esta disciplina todos los campos que bien han sido omitidos total o parcialmente en los estudios de las otras ciencias sociales.

\section{b. Mercado de trabajo y perfil de egreso de la carrera de Sociología}

De las reflexiones de algunos de los/as entrevistados/as se desprende, por una parte, que actualmente no debería de imponerse un perfil determinado, por lo que el o la estudioso/a de lo social, societal y sociológico se desenvuelve en múltiples campos profesionales: Puede desempeñarse como académico/a, investigador/a, funcionario/a de la administración pública, consultor, integrante 
de una Organización No Gubernamental, entre otros, lo cual indica que existe un campo profesional muy amplio en el cual el/la profesional de la Sociología es un interlocutor más que un/a especialista, alguien que propicia el diálogo entre diferentes partes interesadas y afectadas en un punto determinado.

El conocimiento se ha transformado en el componente fundamental de las relaciones de poder y sociales entre los Estados, las clases, los individuos. El conocimiento se ha convertido en la principal fuente de producción en las sociedades modernas y los Estados-Nación. En todo caso, se necesitaría un/a egresado/a con sólidos conocimientos teóricos (para la investigación y el ejercicio de funciones públicas) y metodológicos (capacidad de resolución de problemas) y que sea comprometido y sensible, con un alto sentido de responsabilidad y de ética frente a los problemas más relevantes de nuestra realidad nacional. Un/a profesional con una sólida formación, pero flexible a los cambios que necesariamente tiene que enfrentar sería el perfil deseable o ideal de un/a Sociólogo/a en la actualidad.

Por otra parte, se resalta que ninguna formación profesional puede responder a todas las demandas coyunturales del mercado laboral y más aún cuando se trata de la formación de pregrado. Persiste la inquietud entre el estudiantado de Sociología sobre su futuro profesional, pero obviamente no podemos hacer de ésta un simple conjunto de técnicas y perder la perspectiva crítica-teórica. Algunas de las inquietudes para el ejercicio profesional en los campos específicos se podrían resolver a través de los estudios de cuarto nivel ofrecidos no sólo por las Escuelas de Sociología ${ }^{9}$ sino por otras disciplinas de diferentes escuelas de las universidades.

La mayoría señala que actualmente existe un desencuentro entre el perfil académico y profesional, entre el perfil ideal y el perfil real del/la sociólogo/a. Sin embargo, ante el desafío de los

9 Ninguna de las universidades venezolanas ofertan postgrados de Sociología. 
cambios continuos y de las nuevas exigencias de la realidad social se puede deconstruir y reconstruir un perfil que requeriría de un debate y diálogo con la comunidad de especialistas, académi$\cos /$ as y actores/as que realizan actividades concretas en el país o fuera de él para diseñarlo en función de los requerimientos del entorno como del estado del arte de las ciencias sociales.

En resumen, el perfil del/la sociólogo/a debe ser el de un/a profesional con una alta capacidad crítica (esto lo proporciona toda la base teórica de la carrera) y un manejo metodológico. El/ la profesional de Sociología debe tener conocimiento del debate teórico y metodológico universal, regional y nacional para construir respuestas frente a problemas concretos locales, regionales y nacionales, etc.

Las Escuelas de Sociología en Venezuela deberían egresar, según los/as entrevistados/as, un profesional con la capacidad de:

- Identificar, comprender y explicar las teorías sociales clásicas y contemporáneas.

- Identificar y aplicar los métodos cuantitativos y cualitativos y las técnicas de investigación social.

- Diseñar y ejecutar proyectos de investigación académica y estudios sociales aplicados.

- Identificar, comprender y explicar los procesos sociopolíticos, económicos y culturales de la realidad nacional.

- Diseñar, planificar, coordinar, ejecutar y evaluar políticas públicas y programas sociales.

- Participar y conducir equipos de trabajos multidisciplinarios $\mathrm{y}$ transdisciplinarios.

c. Relación existente entre el mercado de trabajo (administración pública y privada) y las Escuelas de Sociología de Venezuela

Algunos de los/as entrevistados/as consideraron que la relación entre el mercado de trabajo y las Escuelas de Sociología es casi inexistente, por ello la importancia de rediseñar actualmente el 
perfil de Sociología. Las Escuelas de Sociología como las Universidades en general no han logrado establecer una relación efectiva con el sector público y privado, lo que dificulta la inserción de personal calificado en nuestra área de conocimiento. En los planes de estudio están incluidas diversas modalidades de trabajos acreditados, a través de pasantías y de las unidades de extensión y servicio a la comunidad, a fin de establecer vínculos con la realidad nacional a través de organismos extrauniversitarios, participación en proyectos de investigación de interés social, participación en programas de entrenamiento profesional bajo las formas de pasantías y demás modalidades. Sin embargo, existen relaciones tácitas entre algunas Escuelas de Sociología y la administración pública y privada, las cuales deben ser fortalecidas a través de múltiples vías y estrategias, a fin de afianzar intercambios formalizados, con prioridades y objetivos claros y fortalecer el desarrollo de la disciplina.

Como bien se destaca en las reflexiones, no es suficiente que el o la estudiante tenga conocimiento de los procesos sociopolíticos, económicos y culturales del país, es igualmente importante que a lo largo de su formación las Escuelas le proporcionen un acercamiento permanente con las instituciones públicas y privadas, a través de la firma de convenios de intercambio, cooperación, realización de consultorías, asesorías y proyectos aplicados que lleven a cabo las escuelas mediante el financiamiento o auspicio de estas instituciones.

Generalmente suele ocurrir que el/la sociólogo/a una vez que egresa de la Universidad experimenta un profundo desencuentro entre su formación académica y el mercado de trabajo, lo cual obedece al divorcio que existe, a lo largo de su formación, entre los procesos cognitivos y la aplicación de sus conocimientos. Adicionalmente, sucede que las instituciones desconocen las áreas en las cuales el profesional de Sociología puede serles útil, generando la subutilización, invisibilización o la exclusión de este profesional del manual de cargos. 
La Sociología en Venezuela. Una mirada a la disciplina y profesión desde dos generaciones - Mari Carmen Pérez R. y Carmen Teresa García

En líneas generales, los/as entrevistados/as reconocen que las relaciones entre las Escuelas de Sociología y el mercado de trabajo no son del todo satisfactorias, lo cual redunda en la dificultad de los/as egresados de estas escuelas para ubicarse en el mercado laboral. Para que estas relaciones fortalezcan el desarrollo de la Sociología en Venezuela y, por ende, se incremente la demanda de sociólogos/as es imprescindible que las Escuelas aborden la Sociología en constante vinculación con la realidad social y las necesidades de la sociedad venezolana de estos tiempos.

\section{d. La Sociología y su relación con otras disciplinas ${ }^{10}$}

Las reflexiones de los/as entrevistados/as coinciden en afirmar que la Sociología desde sus orígenes trasciende las fronteras disciplinarias, tiene muchas relaciones con diferentes disciplinas, por lo que ha sido una ciencia no sólo interdisciplinaria sino multidisciplinaria y, en la actualidad, transdisciplinaria. Las teorías de Marx, Weber, Durkheim, Parsons, Goffman, Elias, Adorno, Giddens, Morin, entre otros, siempre encontraron y encuentran en desarrollos teóricos distintos de su disciplina una fuente inagotable de conocimiento (Biología, Economía, Política, Epistemología, Filosofía, Cibernética, etc.).

"Se podría decir que los/as sociólogos/as son los enciclopedistas de nuestro siglo. En este sentido, constantemente buscan en campos distintos de reflexión y trabajan en asociaciones con profesionales diversos. No debe existir una separación epistemológica verdadera entre las Ciencias Sociales: ¿cómo podríamos trazar una frontera entre la Historia y la Sociología? De hecho los

10 Entre los pasos hacia la creciente cooperación y coordinación se pueden definir los siguientes: La Disciplinariedad (Especialización aislada), La Multidisciplinariedad (Sin cooperación), La Pluridisciplinariedad (Cooperación sin coordinación), La Cruzdisciplinariedad (Rígida polarización hacia un concepto específico monodisciplinario), La Interdisciplinariedad (Coordinación mediante conceptos de nivel superior) y la Transdisciplinariedad (Coordinación multinivelada del sistema total educación/innovación). Véase Antonio De Lisio. 1999. "Las insuficiencias de la ciencia analítica y la transdisciplinariedad" en RELEA, Nº 
Clásicos realizaban un trabajo sistemático con múltiples herramientas provenientes de todos los campos del saber".

En la actualidad, las fronteras reconocidas de las disciplinas están cada vez más en discusión, el término interdisciplinario está en desuso porque supone la existencia de fronteras entre los saberes desarrollados por las disciplinas. Por el contrario la transdisciplinariedad reconoce la cooperación horizontal entre los saberes, superando las concepciones defensoras de los pequeños feudos de conocimientos y las implicaciones que para el avance de las Ciencias Sociales y Humanas esto ha significado. En este sentido la Sociología es el centro y es partícipe simultáneamente, de otros centros del quehacer cognitivo académico y de los estudios aplicados. La participación de la Sociología en estos centros de saberes no sólo incluye a las disciplinas que integran las Ciencias Sociales, Humanas y Políticas, sino también a las Ciencias Naturales, la biología, la medicina, la ecología, entre otras.

Las ciencias sociales son interdisciplinarias, multidisciplinarias y transdisciplinarias; se requieren unas a otras, su espacio de conocimiento y práctica va más allá de los estrechos límites de cada disciplina. El mercado laboral para la ciencia social es inmenso: desde aquellos espacios institucionales de diversa índole donde se les requiere para actividades de gestión, planificación, coordinación, supervisión, programación y evaluación de proyectos; diseño y evaluación de políticas públicas y en el ámbito del sector privado, hasta los espacios académicos: docencia, investigación. La realidad social exige múltiples requerimientos, en distintas áreas (sociales, políticas, culturales, etc.) y aquellos / as que se formen en Sociología y Antropología, por ejemplo, requieren de conocimientos integrados que les permitan atender la complejidad social.

"La Sociología debe ser transdisciplinaria, lo cual beneficiaría al profesional de sociología en el campo laboral; su relación con el mercado de trabajo pudiera ser fructífera si y sólo si la Sociología forma a un profesional con habilidades múltiples, 
flexibles, con una capacidad de reflexión, de crítica, con un alto nivel teórico y metodológico. La producción de conocimientos de la Sociología supone el desarrollo de alianzas transdisciplinarias que permitan incorporar los diferentes enfoques aportados por otras disciplinas".

Los/as profesionales de la Sociología deben estar en capacidad de participar en equipos transdisciplinarios y establecer relaciones, sin que ello suponga que sus conocimientos, habilidades y destrezas no puedan identificarse y hasta desaparecer como disciplina. Cada vez más es un reto para las organizaciones del siglo XXI transformarse en "organizaciones inteligentes, productivas y competitivas" (públicas, privadas e incluso las universidades y centros e investigación) e incorporar a sus procesos la mayor capacidad de saberes y, por lo tanto, fortalecer los espacios de aprendizaje permanente.

\section{e. Problemas y desafíos de la Sociología}

Las Ciencias Sociales constituyen un área muy variada, compleja y diversa, por lo que existen desacuerdos acerca de ¿qué tipo de ciencia social es posible?, ¿debería tener o no un objeto de estudio determinado con sus métodos y teorías particulares? Sin embargo, la discusión se orienta en la posibilidad de que tanto la Sociología como las demás disciplinas sean concebidas en la actualidad como un área de conocimiento única, por lo que es necesario desarrollar trabajos transdisciplinarios que cuestionen las fronteras artificiales entre los campos de conocimientos.

Entre algunos de los principales problemas se encuentran la incapacidad para dar respuestas a las demandas de la sociedad en lo que atañe al campo del conocimiento sociológico. Superar la ausencia de elaboraciones teóricas que comprendan y vislumbren salidas frente a la crisis y los procesos de transición de las sociedades latinoamericanas en general. La falta de una sensibilidad, compromiso y ética ante la realidad social. El desencuentro 
entre la formación académica recibida y el mercado de trabajo, lo cual obedece a la desarticulación, que ha existido en su formación, entre los procesos cognitivos y la aplicación de los conocimientos y las nuevas políticas sociolaborales de flexibilidad del trabajo.

Entre algunos de los desafíos de la Sociología para el siglo XXI apuntan en primer término a buscar las vías para la superación de las dificultades antes señaladas. Otro gran desafío que confronta la Sociología es la garantía de su permanencia histórica en las Ciencias Sociales, su importancia y utilidad para comprender y actuar sobre las sociedades complejas, en transformación continua y cada vez más excluyentes de la condición humana en sentido integral. Su renovación y permanencia como disciplina que se ocupa del estudio de las relaciones entre los sujetos y la sociedad, pasa necesariamente por dilucidar sobre las preguntas clásicas que le sirvieron de fundamento y dar respuesta a las nuevas interrogantes que emergen de los conflictos y las contradicciones sociales actuales.

En fin, el perfil de egreso del sociólogo/a plasmado en los documentos analizados nos lleva a concluir que a nivel teórico no existen discrepancias entre las diferentes instituciones, que con escasos matices, definen el perfil "ideal" de este profesional, sin embargo cuando abordamos a los profesionales en ejercicio (docentes de la Escuelas de Sociología, investigadores/as, funcionarios/as públicos y privados, entre otros) existe un acuerdo en la definición del objeto de estudio, la vinculación con otras disciplinas, mas no en la definición del perfil académico y profesional, resaltando el desencuentro de ambos perfiles -el del mercado laboral, caracterizado por la flexibilización- y el de las Escuelas de Sociología. 


\section{Una mirada a la Sociología como profesión desde los Sociólogos/as egresados/as en los 90. Resultados de una investigación cuanti-cualitativa}

\section{a. Los datos demográficos de 62 profesionales de sociología (24 hombres y 38 mujeres): ${ }^{11}$}

- La población estudiada de profesionales de Sociología egresados(as) de la década de los 90 es muy joven, se concentra en los grupos etarios entre los 23 a 36 años, predominando el sexo femenino y en un alto porcentaje solteras.

- El nivel de instrucción de los padres y las madres es bajo, ubicándose en sectores trabajadora y asalariada, mientras en sus hijas/os -las/os egresados/as- se revirtió esta realidad, lo que indica que hubo una movilidad social ascendente.

\section{b. De los estudios realizados}

- La mayoría de los(as) egresados/as de la UNELLEZ y de LUZ se inclinaron hacia la Sociología Política y Sociología Industrial (Gerencial, Empresarial y del Trabajo) como línea de investigación del primer intento de investigación formal como es la tesis para optar al título. El problema político sigue siendo todavía el tema de discusión de las tesis, predominando en la Sociología Política los profesionales hombres y en la Industrial las profesionales mujeres.

- La mayoría de los/as egresados/as tuvieron como fuente de ingreso la ayuda familiar para la realización de sus estudios de pregrado.

- La mitad de los/as profesionales encuestados/as realizaron pasantías al final de su carrera como opción (salvo LUZ que no la contempla) y la mayoría fueron las profesionales mujeres.

11 Estos datos del proyecto de investigación en cuestión, fueron también parte del trabajo especial de grado del Br. Eduard Chacin (2003) para optar al título de sociólogo de la LUZ del cual fuimos tutoras, a la vez que él fue asistente de investigación durante el año 2001. 
- Solo 32\% del total ha realizado o realiza postgrado y la mayoría son mujeres y están ubicadas en el Estado Barinas y Mérida. El mayor porcentaje del total es el área de Gerencia, dato que por una parte se vincula y le da continuidad a la tesis de pregrado pero, por otra, es que en el país y en la región Occidental, no hay postgrado en Sociología y las ofertas que existen están orientadas hacia esta especialidad y en general por el sector privado. Del grupo de egresados/as que ha realizado postgrado sólo 3 (5\%) han participado en congresos y uno (1) en investigación y una (1) tiene publicaciones. Datos que evidencian las características de los postgrados cursados, que no contribuyen, pensamos, al desarrollo teórico-metodológico de la Sociología como disciplina y como profesión.

- En relación al interés que motivó a la población encuestada a estudiar Sociología, la mayoría (42\%) se inclinó hacia el Interés sobre la Profesión y un $24 \%$ hacia el interés Intelectual. La mayoría considera que la Sociología les ofrece las herramientas necesarias para cubrir sus inquietudes y para ejercer en el campo laboral.

\section{c. Situación Laboral}

- La mayoría ejercen en las instituciones públicas (89\%). Es la Administración Pública, con Programas Sociales que requieren de sus servicios como profesional, la que representa el mercado de trabajo para el/la sociólogo/a. El 97\% de las mujeres sociólogas trabajan en la administración pública y el 100\% de las desempleadas son mujeres. El 83\% de los sociólogos ejercen en la administración y el resto (hombres y mujeres) ejercen en instituciones privadas.

- $\quad$ El 16\% gana casi el sueldo mínimo, 32\% entre 200 y 400 mil $\mathrm{Bs}^{12} 15 \%$ entre 400 y $600,{ }^{13} 13 \%$ entre 600 y 800 mil Bs y un

12 Lo que equivale, para el año 2001 en que fue recogida la información, a aproximadamente 500 \$ (1 dólar=800 Bs).

13 Loqueequivale, para el año 2001 en que fue recogida la información a aproximadamente 750 \$ (1 dólar= 800 Bs). 
grupo mínimo (8\%) con ingresos superiores a $800 \mathrm{mil}$, es decir, oscilaba entre 500 a $1000 \${ }^{14}$. A pesar, de que el Colegio Nacional de Sociólogos y Antropólogos de Venezuela, ha regulado a través de su ley (Art. 8) "que en ningún caso el salario mínimo debe ser menor que los obtenidos por profesionales de otros gremios, ni por debajo del que establezca el Ejecutivo Nacional para los organismos públicos". Las cifras evidencian que más de la mitad de los/as egresados/as están subpagadas/os mostrando la tendencia de que flexibilidad salarial se impone frente a la ley profesional.

- Solo el 16\% del total ejercen el cargo de sociólogo/a, 13\% el cargo de Coordinador/a, 13\% de Docente, $8 \%$ el cargo de Auxiliar de Investigación, 6\% (c/u) los cargos de Trabajadores/as Sociales, Planificadores/as y $3 \%$ (c/u) en cargos administrativos, promoción social y el resto representan el 2\% cada uno.

Sólo el 30\% del total ejerce cargos de sociólogo/a, Auxiliar de Investigación, Planificador/a en el que se podrían vincular con el perfil ideal que está plasmado en los planes de estudio de la Escuelas de Sociología, el resto (70\%) se desempeña en cargos genéricos (polivalentes), pues su quehacer se perfila en función de las necesidades y lineamientos de la institución empleadora; esto reafirma la tendencia de la flexibilidad laboral que caracterizó la década de los 90 .

El 58\% de los/as egresados/as afirman que hay una relación entre el empleo y los estudios de Sociología y casi un 30\% expresan lo contrario (ninguna y alguna relación). Esto conduce a pensar que por la diversidad de cargos que desempeñan los/ as egresados/as, pareciera, los planes de estudios, no se corresponde con el mercado de trabajo, sin embargo, consideramos que les ofrece conocimientos y herramientas metodológicas básicas para enfrentarse ante tal diversidad, a tal punto que esta mayo-

14 En 2003 el \$ oficial estaba en 1600Bs y en 2006 está en 2150 Bs. 
ría (58\%) consideran que si hay adecuación total entre estudios y empleo. Por tal motivo, un $84 \%$ afirman que están satisfechos con su carrera. Muchos más de los que señalan que hay una relación estudios y empleo.

- En relación a las asociaciones en las que participan los/as sociólogos/as, 19\% participan en sindicatos y asociaciones profesionales, 23\% participa en la organización gremial (sólo existe en el estado Zulia), 53\% no contestó o no participa en ninguna de las asociaciones. Y todo esto, a pesar de que los planes de estudio de las escuelas forman a sus egresados/as como organizadores/as sociales y como consecuencia, los/as profesionales de la Sociología se encuentran desprotegidos gremialmente (como hemos visto con los salarios -flexibilidad salarial-, la casi desaparición del cargo de Sociólogo/a en la Administración Pública que representa su mayor fuente de empleo, la flexibilidad del empleo expresada en la trayectoria de la experiencia laboral de los/as egresados/as en la década de los 90) y como disciplina se ve afectada por las limitaciones que esto genera.

\section{A manera de conclusiones}

Finalmente queremos, más que brindar conclusiones acabadas, recoger algunos de los aspectos más relevantes que se desprenden del trabajo realizado, a fin de brindar elementos para la discusión sobre la evaluación de la Sociología como disciplina y como profesión (práctica/quehacer de los profesionales de sociología). Consideramos que con base en la discusión que se pueda generar a partir de estos y otros planteamientos no presentes en este trabajo, es necesario pensar en la posibilidad de reconstruir un perfil, un plan de estudios y el ethos del profesional de Sociología en los tiempos actuales. 


\section{Una mirada sociológica a la sociología como disciplina y profesión. ¿Sociólogos/Sociólogas y Sociología sin fronteras?}

De los resultados de la investigación se podría afirmar que existe un desencuentro entre el perfil académico y el profesional, una invisibilización de la disciplina y la profesión en el mercado de trabajo en tiempos de globalización neoliberal y, finalmente, queremos dejar abiertos para la discusión los desafíos que tiene la Sociología como disciplina y profesión ante la transdisciplinariedad que también la invisibiliza y/o la desaparece como tal.

¿Cuáles desafíos tiene que enfrentar? Desde nuestra mirada la Sociología debe:

- Repensar las crisis de los paradigmas como un compromiso impostergable

- Ir descifrando la complejidad social. Construcción del saber multidimensional e integrado de la sociedad con miras, multi, inter y transdisciplinarias. La comprensión de la complejidad del mundo social de hoy, en sus múltiples dimensiones, además de la diversidad de saberes y prácticas. Pero ¿esta transdisciplinariedad no la desaparece o invisibiliza?

- El desafío de la Sociología ante el proceso de globalización o globocolonización ${ }^{15}$. Los retos de la sociología ante la compleja realidad social existente, frente a un mundo que se globaliza pero que excluye a las mayorías.

- Responder a las incertidumbres que generan los tiempos de transición y de las constantes transformaciones de la realidad social (Caso Venezuela): La incertidumbre de lo real, del conocimiento, del mundo cambiante.

- Repensar y revalorizarnos, en un contexto de transdisciplinariedad, como disciplina y profesión.

- Repensar y afrontar los problemas de la profesionalización

15 Véase el Artículo de MariCarmen Pérez. 1998. “El desafío de la sociología ante el proceso de globalización", en FERMENTUM (Revista Venezolana de Sociología y Antropología), Año 8, N²1, Enero-Abril, GISAC-ULA, Mérida, Venezuela. 
de la Sociología en el mercado de trabajo actual que la flexibiliza y hasta la desfigura, en el sentido de que elimina las denominaciones propias de los cargos de sociólogo/a por los genéricos (polivalentes), como se pudo constatar en la muestra estudiada, lo cual reafirma la invisibilización de la profesión en esta década.

- Repensar el quehacer sociológico y su responsabilidad intelectual en esta coyuntura tan importante para América Latina y Venezuela en particular, donde lo social, lo societal y lo sociológico cobran importancia en el contexto sociopolítico y socioeconómico actual.

- Revisar, repensar el compromiso (con quién y para qué) y la ética del profesional de Sociología.

- Reflexionar y redefinir el perfil y el oficio sociológico (definición del perfil actual, ideal, real de los/as egresados/as de Sociología; el perfil profesional que demandan los organismos públicos y privados que pareciera que es el que impera frente al académico).

- Revisar los contenidos temáticos en los planes de estudios y las técnicas y conocimientos que debe dominar el actual profesional de Sociología.

- Reflexionar sobre la vinculación de la teoría y la praxis, entre teoricismo y empirismo.

- En fin, afrontar el desafío del pluriparadigmatismo, del eclecticismo, como tendencias de la Sociología.

\section{Bibliografía}

Casarini, R. (1999). Teoría y diseño curricular, México: ITESM, Edit. Trillas, Universidad Virtual.

Castro, Gregorio Antonio (1988). Sociólogos y Sociología en Venezuela, UNESCO, Fondo Editorial Tropykos, Caracas.

(1998). "La Sociología frente al próximo milenio", en Espacio Abierto (Cuaderno Venezolano de Sociología), Universidad del Zulia, Vol.7, N³, Septiembre-Diciembre, Maracaibo-Estado Zulia, Venezuela. 
La Sociología en Venezuela. Una mirada a la disciplina y profesión desde dos generaciones - Mari Carmen Pérez R. y Carmen Teresa García

Consejo Nacional de Universidades (CNU), Oficina de Planificación del Sector Universitario (OPSU). (2002). Oportunidades de Estudio en las Instituciones de Educación Superior de Venezuela, Caracas.

Chacin S. Eduard (2003). La práctica profesional de sociólogos/as en el Occidente del país. Trabajo especial de grado Faces, Escuela de Sociología LUZ, Maracaibo.

De Lisio, Antonio (1999). “Las insuficiencias de la ciencia analítica y la transdisciplinariedad como alternativa" en RELEA, $\mathrm{N}^{\circ} 7$, Caracas.

García, Carmen Teresa y otros (1991). “La investigación socioantropológica en Venezuela. Una aproximación a través de los tres Congresos Nacionales de Sociología y Antropología (1981-1982-1990)" en FERMENTUM (Revista Venezolana de Sociología y Antropología), Año 1, №2, GISAC-ULA, Septiembre-Diciembre, Mérida, Venezuela.

García, Carmen Teresa (1998). "El quehacer de la sociología en tiempos de crisis", en FERMENTUM Año 8, №21, Grupo de Investigación en Socioantropología de la Ciudad de la Universidad de Los Andes (GISAC-ULA), Enero-Abril, Mérida, Venezuela.

García, Carmen Teresa y MariCarmen Pérez (2000). "Repensar la crisis de los paradigmas: Un compromiso impostergable", en FERMENTUM Año 10, N²7, Grupo de Investigación en Socioantropología de la Ciudad de la Universidad de Los Andes (GISACULA), Enero-Abril, Mérida, Venezuela.

Giddens, A. (2000). En defensa de la Sociología, Madrid, Edit. Alianza.

Glazman, N. (1994). "Situación actual de las universidades públicas. Perfil del egresado y curriculum", en Grupo de Investigación en Socioantropología de la Ciudad de la Universidad de Los Andes (GISAC-ULA). 1996. "Investigar y publicar: Una relación indispensable", FERMENTUM (Revista Venezolana de Sociología y Antropología), Año 6, N¹5, GISAC-ULA, Enero-Abril, Mérida, Venezuela.

Grupo de Investigación en Socioantropología de la Ciudad de la Universidad de Los Andes (GISAC-ULA) (1993). XIX Congreso de la Asociación Latinoamericana de Sociología, 30 de mayo al 04 de junio, 1993, Caracas, Venezuela, FERMENTUM (Revista Venezolana de Sociología y Antropología), Año 3, Nos. 6 y 7 , GISAC-ULA, Enero-Agosto, Mérida, Venezuela. 
Guédez, Víctor (2000). Definiciones (Mimeo), Taller sobre Educación y Evaluación Educacional, FOULA, Universidad de Los Andes, Mérida.

Infestas, A. y Lambea, M. (1997). Los intereses de la sociología actual, Proyecto A. Ediciones, Barcelona, España.

Ley de Ejercicio Profesional de la Sociología y la Antropología, República de Venezuela, Caracas, 1998.

Morín, Edgar (1995). Epistemología de la complejidad, en Dora Fried Schnitman (Edit.) Nuevos paradigmas, cultura y subjetividad. Paidós, Buenos Aires.

(2000). Los siete saberes necesarios a la educación del futuro, UNESCO/IESALC, FACES/UCV, CIPOST, Caracas.

Oficina Central de Personal (1990). Manual Descriptivo de clases de cargos, Oficina de Capacitación Profesional, Caracas.

Pensum de la Escuela de Ciencias Sociales (Sociología), Facultad de Ciencias Económicas y Sociales de la Universidad Católica Andrés Bello (UCAB), Caracas, junio, 1993.

Pensum de la Escuela de Sociología, Facultad de Ciencias Económicas y Sociales de la Universidad Central de Venezuela (UCV), Caracas, junio 1995.

Pensum de la Escuela de Sociología, Facultad de Ciencias Económicas y Sociales de La Universidad del Zulia (LUZ), Maracaibo-Estado Zulia, marzo 1995.

Pensum del Programa Sociología del Desarrollo, Escuela de Sociología de la Universidad Nacional Experimental de Los Llanos Occidentales "Ezequiel Zamora” (UNELLEZ), Barinas-Estado Barinas, mayo 1995.

Pérez R., MariCarmen (1998). "El desafío de la Sociología ante el proceso de globalización", en FERMENTUM (Revista Venezolana de Sociología y Antropología), Año 8, №21, GISAC-ULA, eneroabril, Mérida, Venezuela.

(2001). Estado y Empleo en el países miembros del G3 en la década de los 90. Tendencias y Cambios. Trabajo de Grado de Magíster en Cs. Políticas ULA.

Ritzer, George (1996). Teoría Sociológica Contemporánea, McGraw-Hill, Caracas. 
La Sociología en Venezuela. Una mirada a la disciplina y profesión desde dos generaciones - Mari Carmen Pérez R. y Carmen Teresa García

Sabino, Carlos (1986). El Proceso de Investigación, Editorial Panapo, Caracas.

Santana Cova, N. (1997). "El Estado, la globalización del sector agroalimentario y su incidencia en el mercado laboral. El papel de las microempresas agrícolas". En Sociología Política. ULA Mérida, pp. 239-259.

Zamitiz G., Héctor (1995). “Evaluación de la carrera de ciencia política en México: Entrevistas con Especialistas", en Revista Estudios Políticos, Facultad de Ciencias Políticas y Sociales, UNAM, $4^{\text {a }}$.Época, $\mathrm{N}^{\circ} 8$, julio-septiembre, México D.F. 